\title{
Evidence for apoptosis, MMP-1, MMP-3 and TIMP-2 expression and their effect on the mechanical and biochemical properties of fresh viable knee medial meniscal allografts and autografts in the rabbit
}

\author{
Tomasz J. Zwierzchowski ${ }^{1}$, Olga Stasikowska-Kanicka², Jolanta Janus ${ }^{3}$, Włodzimierz Konecki, \\ Marian Danilewicz ${ }^{2}$, Jarosław Fabiś ${ }^{1}$
}

\begin{abstract}
${ }^{1}$ Arthroscopy and Sports Traumatology Clinic, Orthopaedic Department, Medical University of Lodz, Poland

2Department of Nephropathology, Medical University of Lodz, Poland

${ }^{3}$ Department of Pathophysiology, Medical University of Lodz, Poland

${ }^{4}$ Department of Fibre Physics and Textile Metrology, Technical University of Lodz, Poland
\end{abstract}

Submitted: 27 December 2010

Accepted: 31 July 2011

Arch Med Sci 2012; 8, 4: 724-732

DOI: 10.5114 /aoms.2012.30297

Copyright (c) 2012 Termedia \& Banach

\begin{abstract}
Introduction: The study sought evidence for apoptosis, the expression of MMP-1, MMP-3 and TIMP-2 and their effect on the mechanical and biochemical properties of rabbit fresh knee medial meniscal grafts in a 6-month follow-up.

Material and methods: Forty white male New Zealand rabbits were chosen for the study. The medial meniscus was excised from 28 animals and stored under tissue culture conditions for 2 weeks, following which 14 of them were implanted as autografts and 14 as allografts. When the animals were euthanized, 20 menisci were used for immunohistochemical examinations. Apoptosis (TUNEL method) and MMP-1, MMP-3 and TIMP-2 immunoexpression were estimated semiquantitatively. The other 20 menisci were subjected to biochemical analysis and their degree of elasticity was evaluated.

Results: An increased level of apoptosis $(p<0.05)$ was observed both in allografts $(1.57 \pm 0.98)$ and autografts $(0.86 \pm 0.69)$; no statistical differences existed between them. An increased level of metalloproteinases and TIMP-2 expression was observed only in the allografts $(p<0.05)$. The highest decrease of degree of elasticity and the most significant changes in biochemical composition were observed in allografts $(p<0.05)$.

Conclusions: The studies confirmed the existence of excessive apoptosis in both kinds of fresh viable medial meniscal implants: auto- and allografts. Our results suggest that apoptosis and increased MMP-1 and MMP-3 expression have an adverse effect on the biological properties of implants. The results of experimental studies on humans indicate the need to devise a method of apoptosis inhibition in transplanted menisci to improve long-term results.
\end{abstract}

Key words: apoptosis, metalloproteinases, viable meniscal transplant properties.

\section{Introduction}

At present, little is known both of the occurrence and the level of apoptosis as well as of the expression of tissue metalloproteinases in knee meniscal allografts and their potential effect on biochemical and mechanical properties [1].

\author{
Corresponding author: \\ Tomasz Jacek Zwierzchowski \\ MD, PhD \\ Arthroscopy and Sports \\ Traumatology Clinic \\ Orthopaedic Department \\ Medical University of Lodz \\ 113 Żeromskiego \\ 90-549 Lodz, Poland \\ Phone: +48 509508781 \\ Fax: +48 426393539 \\ E-mail: \\ zwierzchowski.jacek@gmail.com
}


So far, it has been demonstrated in vivo that natural meniscal cells undergo apoptosis and extracellular matrix changes are regulated by tissue metalloproteinases [2-6]. Thus, hypothetically, apoptosis and metalloproteinases should play a significant role in preserving the homeostasis of the implanted meniscus and influence its mechanical properties and biochemical structure.

The presence of an appropriate number of viable proliferating cells producing matrix molecules, together with the activity of metalloproteinases preserving extracellular matrix integrity, are the necessary conditions of a successful implantation [7]. Therefore, recognition of the phenomena taking place in the implanted meniscus, which may lead to changed biological properties of the graft, is of key clinical importance [8-12]. Apoptosis is a highly regulated form of cell death which preserves homeostasis, eliminating unwanted cells [13-15]. Matrix metalloproteinases take part in extracellular matrix degradation, regulate its changes and preserve matrix homeostasis [16, 17]. Increased metalloproteinase expression occurs in degenerative diseases and causes irreversible changes in matrix composition $[4,18]$. The biochemical structure of the meniscus indicates the significant role of collagenase 1 and stromelysin 1 [2]. Tissue inhibitors of metalloproteinases (TIMPs) inhibit their activity, and TIMP-2 demonstrates a number of biological activities which can affect the properties of the implanted meniscus [16-19].

It has been assumed that fresh meniscal grafts stored under tissue culture conditions could potentially shed more light on the hypothetical long-term beneficial effect of extracellular matrix molecules synthesised by viable cells at the moment of implantation [20]. Owing to its ideal size, an autograft will enable biological phenomena occurring in an allograft to be objectivised [8, 11, 12]. It will also allow the assessment of potential possibilities for autografts produced in the near future owing to the progress in tissue engineering and its comparison with an analogous allograft.

The hypothesis of the study was that apoptosis and metalloproteinases may play a crucial role in meniscal grafts' subsistence due to their influence on the biochemical and mechanical properties of the transplants. The aim of the study was to assess the level of apoptosis, the expression of MMP-1, MMP-3 and TIMP-2 as well as the biochemical and mechanical properties of rabbit fresh knee medial meniscal allo- and autografts stored under tissue culture conditions for 2 weeks in a 6-month follow-up.

\section{Material and methods}

\section{Study design}

Forty-six white male New Zealand rabbits, body weight 3500-4000 g, were chosen for the study. All procedures were performed with the consent of the Local Ethical Committee for Experiments on Animals (Number $七 / B D / 254)$. Two study groups were distinguished: 17 animals with allografts and 17 with autografts. The third was a control group of 12 animals which underwent arthrotomy (sham operations). The excised left knee medial menisci (from 34 animals) were stored under tissue culture conditions. After 2 weeks' storage, to replicate clinical conditions, 17 animals were implanted with own autografts and 17 with donor allografts. Six rabbits (3 with autografts and 3 with allografts) developed infective arthritis and were excluded from the study. All animals were euthanised after 6 months, when the implanted grafts were at an advanced stage of rebuilding. Twenty menisci were subjected to immunohistochemical analysis (posterior region) and 20 to mechanical and biochemical analyses (posterior region). The choice of posterior third used for immunohistochemical, mechanical and biochemical tests was based on previous observations by other authors [8-10].

\section{Surgical procedure}

All surgical procedures were performed by the same surgeon. The rabbits were premedicated with intravenous Medetomidine (Dormitor) at a dose of $0.01 \mathrm{ml} / \mathrm{kg}$ b.w. and atropine at a dose of 0.05 $\mathrm{mg} / \mathrm{kg}$ b.w. To maintain anaesthesia, Bioketan (ketamine) was administered at an intramuscular dose of $3 \mathrm{mg} / \mathrm{kg}$ b.w. Atipamezole at a dose of $0.01 \mathrm{ml} / \mathrm{kg}$ b.w. was used to wake the animals. Under aseptic conditions, a skin incision of about $25 \mathrm{~mm}$ was performed on the medial side, and two medial anterior and posterior openings were made in the left joint capsule. The medial meniscus was excised according to the modified Shibuya technique without cutting collateral tibial ligament [21]. The wound was closed in layers. The excised meniscus was placed in a tissue culture bottle Nunc Flasks (NuncDenmark) with Eagle medium (DNEM: Ham F12 (1: 1$)$, Gibco) with the addition of $10 \%$ fetal calf serum (Sigma) and 1\% penicillin, streptomycin and Fungizone (Gibco). The containers were stored in an incubator for 2 weeks, at $37^{\circ} \mathrm{C}$ in an atmosphere of $5 \% \mathrm{CO}_{2}$ and $95 \%$ air. Every 3 days, the bottles and medium were exchanged. From among the menisci prepared in this way, 17 rabbits were implanted with their own menisci into the left knee joint - autografts. The remaining 17 animals were implanted with donor menisci - allografts. The medial meniscus was implanted according to a modified technique of Sommerlath and Gillquist [22]. After each procedure, antibiotic (enrofloxacin) was administered for prophylactic purposes at an intramuscular dose of $10 \mathrm{mg} / \mathrm{kg}$ b.w. every $12 \mathrm{~h}$ for 3 days. After surgery, the animals received butorphanol subcutaneously (0.3 mg/kg b.w.). No plas- 
ter immobilization was applied but the rabbits were allowed to move freely in standard cages. The animals' behaviour was observed systematically. Postoperative wounds healed per primam, except six animals, which were excluded from the study.

After 6 months, the animals were euthanised by administering a lethal dose of Pentobarbital intravenously. The knee joint was opened in the left limb. The medial menisci were carefully excised from all rabbits for further investigation. Twenty menisci (half from each study group and the controls) designated for immunohistochemical examinations were placed in containers with $10 \%$ buffered formalin. The remaining 20 menisci were immediately subjected to mechanical tests and analysis of biochemical composition.

\section{Apoptosis detection}

Apoptosis was evaluated using the TUNEL method. Formalin-fixed, paraffin-embedded tissue sections were mounted onto SuperFrost slides and deparaffinized with xylene, washed with alcohol and transferred to distilled water. Afterwards, a TUNEL Apoptosis Detection Kit (DNA Fragmentation/Fluorescence Staining; No 17-141; Upstate NY) was used according to the instructions of the manufacturer. In each specimen immunofluorescence was recorded semiquantitatively according to Durand et al. in 3-7 adjacent high power fields and the mean grade was calculated [23]. In the select-

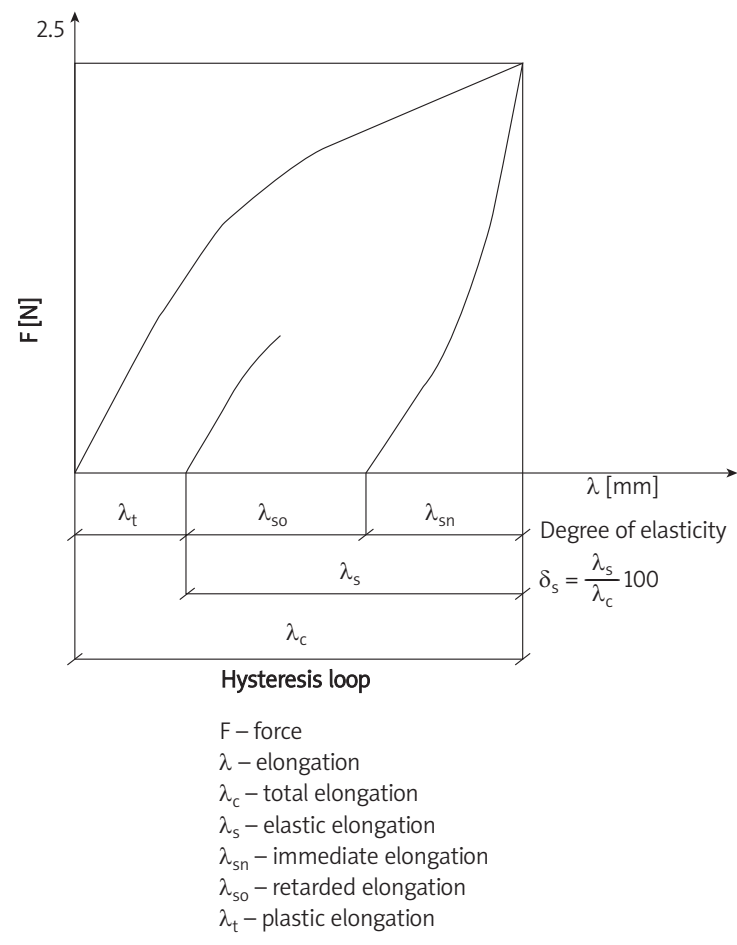

Figure 1. Exemplary hysteresis loop for determination of degree of elasticity ed samples, apoptosis was confirmed by evaluating its morphological exponents in $\mathrm{H}+\mathrm{E}$ stained specimens under a light microscope.

\section{Immunohistochemistry}

Paraffin sections were mounted onto SuperFrost slides, deparaffinized, then treated in a microwave oven in a solution of EDTA, pH 8.0 for $20 \min (2 \times$ $360 \mathrm{~W}-5 \mathrm{~min}, 2 \times 180 \mathrm{~W}-5 \mathrm{~min}$ ) and transferred to distilled water. Endogenous peroxidase activity was blocked by $0.3 \%$ hydrogen peroxide in distilled water for $30 \mathrm{~min}$, and then the sections were rinsed with Tris-buffered saline (TBS, DakoCytomation, Denmark) and incubated overnight at $4^{\circ} \mathrm{C}$, with the following mouse monoclonal antibodies: anti-MMP-1 (Chemicon, MAB 3307, Germany, dilution 1 : 200), anti-MMP-3 (Chemicon, MAB 3312, Germany, dilution $1: 250)$ and rabbit monoclonal antibody against TIMP-2 (Chemicon, AB 19029, Germany, dilution 1 : 300). Afterwards, appropriate EnVision + Systems-HRP (DakoCytomation, Denmark) were used, prepared according to the instructions of the manufacturer. Visualisation was performed by incubating the sections in a solution of 3,3'-diaminobenzidine (DakoCytomation, Denmark). After washing, the sections were counter-stained with haematoxylin and cover-slipped. For each antibody and for each sample, negative controls were processed by incubation in the absence of the primary antibody, and were found to always yield negative results.

The staining intensities of MMP-1, MMP-3 and TIMP-2 were recorded semiquantitatively according to Choi et al. in 3-7 consecutive high power fields [24]. Then the mean grade was calculated.

Apoptosis and immunohistochemistry evaluation was performed by a single observer, who was blinded to the experimental groups.

\section{Mechanical test}

The mechanical evaluation of menisci probes was based on a stress-relaxation test [25]. Immediately after excision, the posterior third of menisci were weighed and subjected to a compression test on a floor model INSTRON TT-BM testing instrument. The meniscus was placed in a specially designed metal nonporous matrix with a shape matching the concavo-cuneiform shape of the meniscus, which prevented the specimen shifting laterally during compression. The posterior region of the menisci was compressed with a force of $2.5 \mathrm{~N}$, held for $5 \mathrm{~min}$ and allowed to relax. The degree of elasticity, calculated from elastic and total elongation, was determined from the obtained hysteresis curve (Figure 1). The mechanical parameters were determined based on mechanical properties of rabbit medial meniscus [22]. 


\section{Biochemical evaluation}

Directly after mechanical testing, the probes of menisci were dried, weighed and subjected to biochemical analysis. The water content was calculated by subtracting the dry weight from the wet weight of the meniscus. The colorimetric method described by Woessner was used to assess the hydroxyproline fraction [26]. The total collagen content was derived from hydroxyproline by multiplying its value by a factor of 7.4 as suggested by Ingman et al. [27]. The determination of total sulphated GAG content was based on the method described by Ferndale et al. [28].

\section{Statistical analysis}

All values were expressed as the mean \pm SD (standard deviation). All the variables were treated as measurable ones. The Shapiro-Wilk normality test served as a basis for deciding on a parametric or nonparametric statistical test. In all groups there was no reason to reject the hypothesis that the variable has a normal distribution, except for apoptosis, which does not appear in the control group. The ShapiroWilk normality test results enabled the application of parametric methods in the subsequent calculations. Next Levene's test was conducted, which rejected the hypothesis of homogeneity of variances for apoptosis and immunohistochemistry. In those cases the non-parametric Kruskal-Wallis test was used for the evaluation of statistical differences between experimental and control groups. For the mechanical and
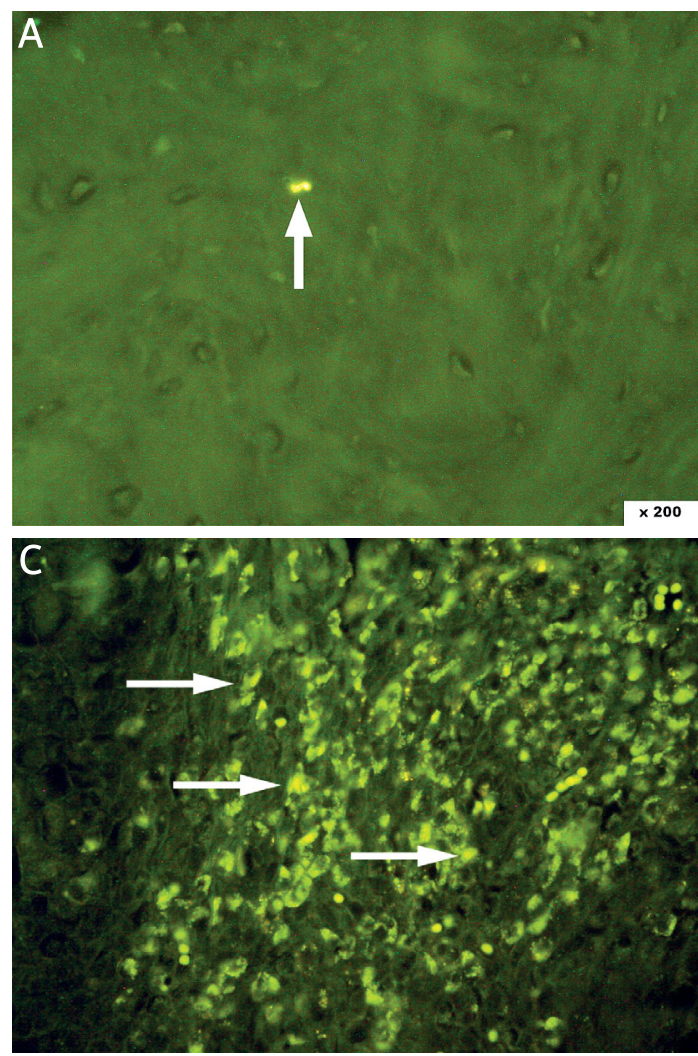

biochemical studies Levene's test results enabled the use of an ANOVA (analysis of variance) test. Value of $p<0.05$ was considered to be the level of statistical significance.

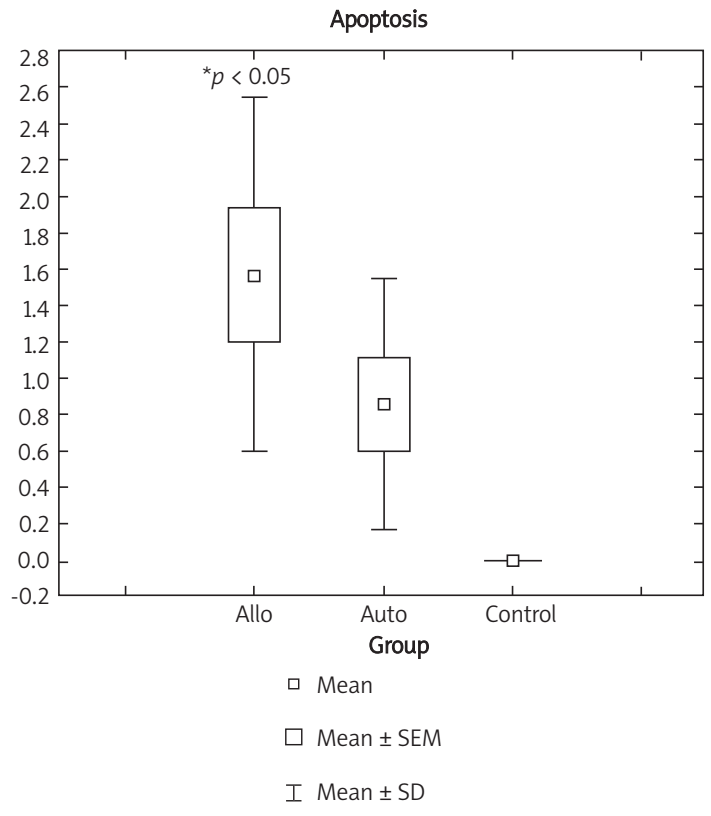

${ }^{*} p<0.05$ between allografts and control

Figure 2. Apoptosis level in allografts (allo), autografts (auto) and control in the posterior part of the meniscus

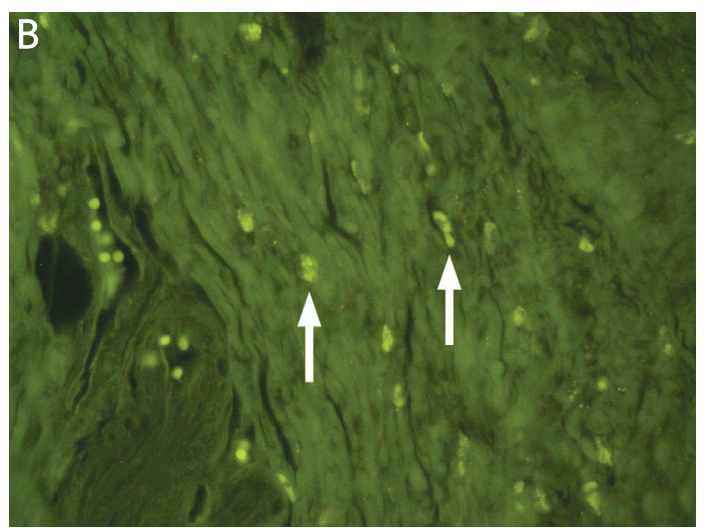

Figure 3. TUNEL staining demonstrating apoptotic cells in the posterior part of the meniscus (arrows). A - Control (artefact) (TIFF); B - autograft (TIFF); C - allograft (TIFF) 


\section{Results}

No histological evidence of rejection or inflammatory reaction was observed in any transplanted menisci. There were no plasma cells or infiltration of lymphocytes and macrophages in the transplant menisci.

\section{Apoptosis analysis}

The TUNEL test demonstrated increased apoptosis both in the group of autogenous grafts (mean: 0.86 \pm 0.69 ) and in the group of allogenous grafts (mean: $1.57 \pm 0.98$ ) (Figure 2). Statistical analysis did not confirm any significant differences between the two study groups. However, a difference $(p<0.05)$ was observed between the allograft group and the control group, in which no apoptosis was found (Figure 3).

\section{Immunohistochemistry}

The mean values of the immunoexpression of MMP-1, MMP-3 and TIMP-2 are given in Table I. The

Table I. The immunoexpression of MMP-1, MMP-3 and TIMP-2 in study groups and control

\begin{tabular}{|lccl|}
\hline Group & MMP-1 & MMP-3 & TIMP-2 \\
\hline Control & $0.15 \pm 0.15$ & $0.22 \pm 0.06$ & $0.26 \pm 0.09$ \\
\hline Autografts & $0.16 \pm 0.19$ & $0.26 \pm 0.30$ & $0.16 \pm 0.19$ \\
\hline Allografts & $2.25 \pm 0.59^{*} \&$ & $2.29 \pm 0.63^{*} \&$ & $1.40 \pm 0.58^{*} \&$ \\
\hline
\end{tabular}

Mean value \pm standard deviation (SD). *Statistically significant between control and allografts $(p<0.05)$. \& Statistically significant between auto- and allografts $(p<0.05)$
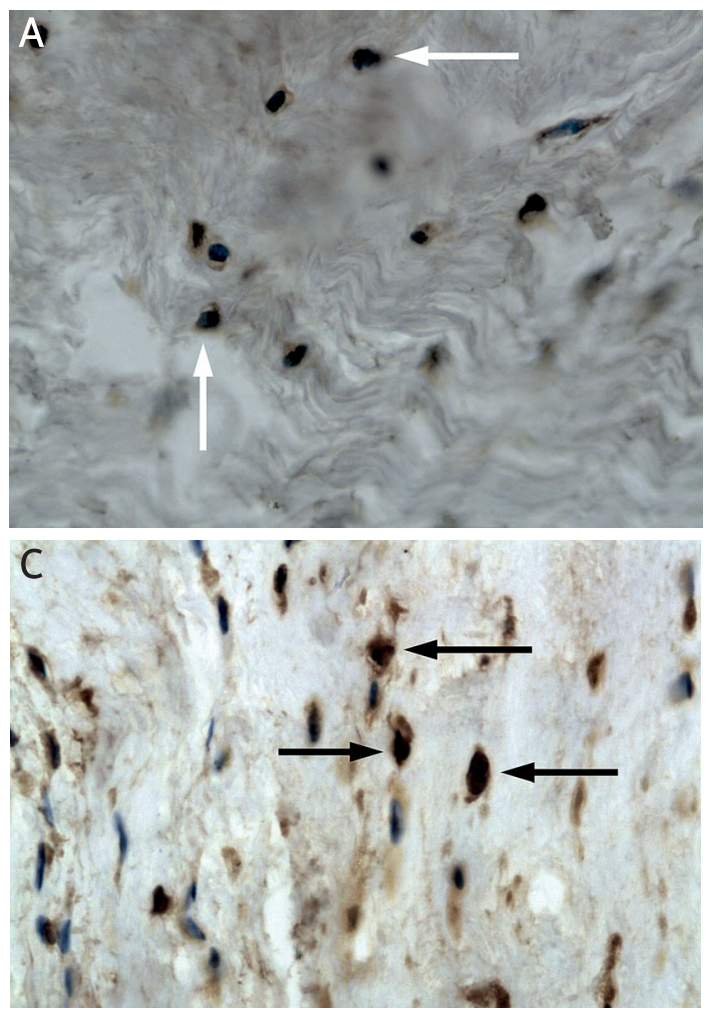

immunoexpression of MMP-1, MMP-3 and TIMP-2 was significantly increased in the allografts, as compared to the autografts and controls $(p<0.05)$ (Figures 4-6). The difference of the MMP-1, MMP-3 and TIMP-2 immunoexpression between autografts and controls was weak and not significant.

\section{Mechanical test}

The meniscal degree of elasticity was seen to be lower in the group of autogenous grafts $(p<0.05)$ (mean: $23.64 \pm 0.80$ ) in relation to the controls (mean: $29.37 \pm 1.03$ ) by a statistically significant difference. The highest decrease was observed in the group of allografts (mean: $17.16 \pm 0.80$ ) with a statistically significant difference between the controls and allogenous grafts $(p<0.05)$ (Figure 7$)$.

\section{Biochemical analysis}

The values of biochemical parameters in the study groups and in the control group are given in Table II.

\section{Discussion}

A review of the literature indicates that this is the first study to demonstrate the presence of apoptosis in knee meniscal grafts. Moreover, the expression of metalloproteinases has been assessed in rabbit fresh knee medial meniscal auto- and allografts. Mechanical and biochemical analyses have enabled the effect of apoptosis and metallo-

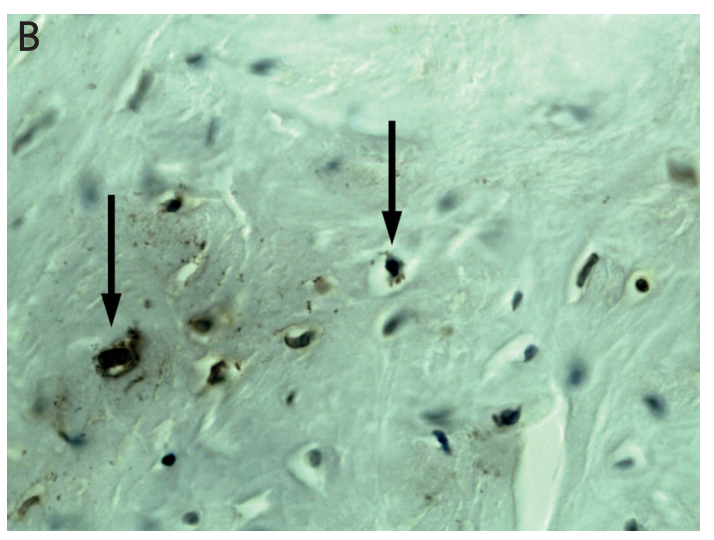

Figure 4. Immunohistochemical staining of MMP-1 in the posterior part of the meniscus (arrows). A - Control (TIFF); B - autograft (TIFF); C - allograft (TIFF) 

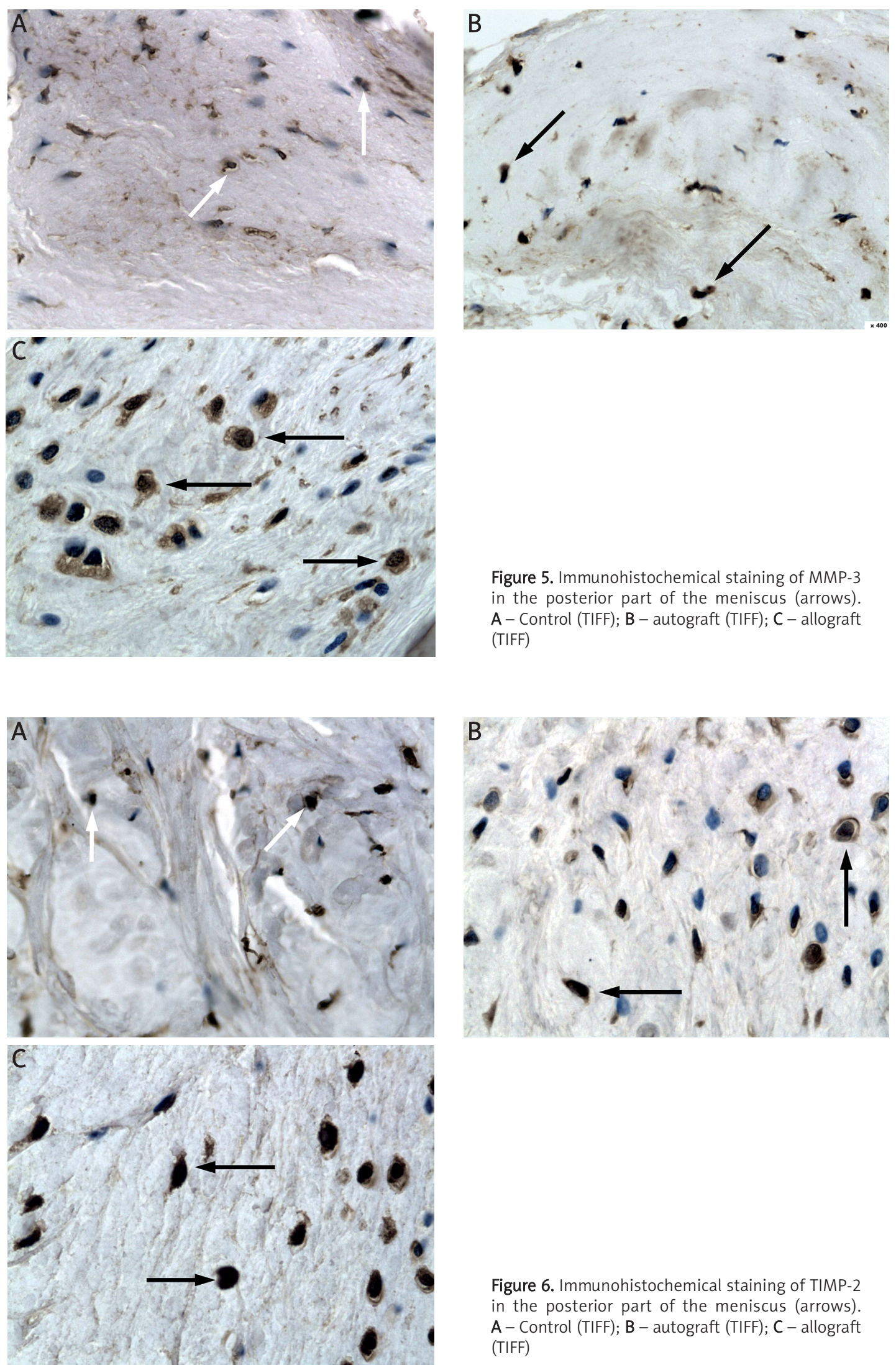

Figure 5. Immunohistochemical staining of MMP-3 in the posterior part of the meniscus (arrows). A - Control (TIFF); B - autograft (TIFF); C - allograft (TIFF)

Figure 6. Immunohistochemical staining of TIMP-2 in the posterior part of the meniscus (arrows). A - Control (TIFF); B - autograft (TIFF); C - allograft (TIFF) 


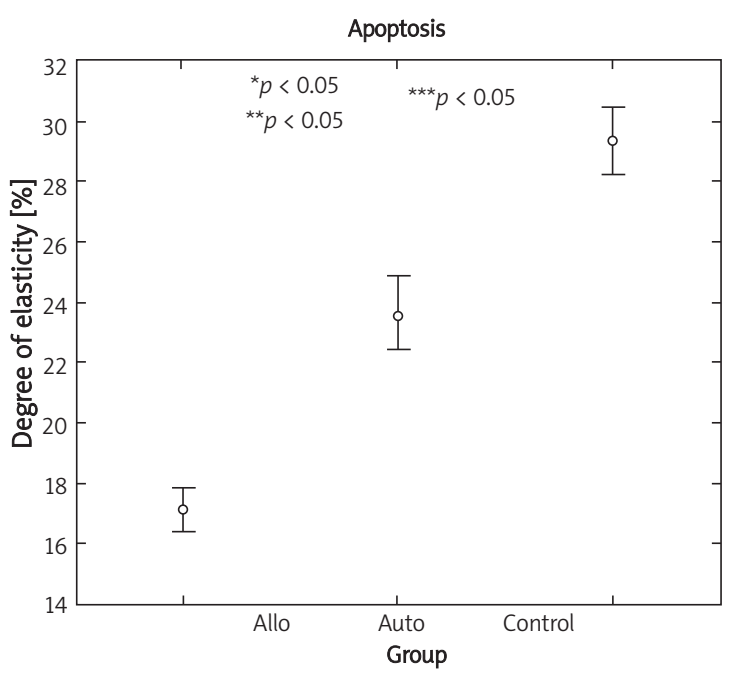

Figure 7. Degree of elasticity (\%) in allografts (allo), autografts (auto) and control

${ }^{*} p<0.05$ between allografts and control, ${ }^{* *} p<0.05$ between allografts and autografts, ${ }^{* * *} p<0.05$ between autografts and control

proteinases on the biological properties of implanted menisci to be assessed, and to be compared with fresh auto- and allografts stored for 2 weeks under tissue culture conditions, with a 6-month follow-up.

Our own analysis using the TUNEL method demonstrated increased apoptosis in both groups of auto- and allografts. A lack of statistical differences between both groups was observed. Apoptosis is of clinical importance and its dysregulation occurs in an early stage of degenerative disease in the meniscus [4, 5]. Apoptosis is rarely detected in a normal native meniscus [2]. This is consistent with our observation that it was not found in our control group, which suggests that arthrotomy does not induce apoptosis, any increase of which is associated exclusively with the meniscal implant.

Our studies suggest that an early stage of degenerative disease is observed in fresh knee medial meniscal grafts in rabbits, which questions the value of this implant. The previously demonstrated relationship between apoptosis and decreased population of cells in a native meniscus can be assumed to be true also for meniscal implants [4]. The increased apoptosis in auto- and allografts revealed by this study may explain the decreased population of cells in meniscal implants observed by other authors [8-12].

The unsuccessful results are related to the hypocellularity or acellularity of the implanted meniscus [29]. Our studies have confirmed that excessive apoptosis of the implanted meniscus cells worsens the results of the implantation.

The observations of Le Graverand about the effect of apoptosis on the biochemical composition of the native menisci support our study [3]. In both study groups, a decrease was observed in the content of proteoglycans and an increase of water content, which lead to a decrease of elasticity. Our observations partly coincide with the findings of Jackson regarding the altered biochemical composition of implanted menisci in goats in a 6-month follow-up. Contrary to Jackson, we did not detect an increase but in fact found a decrease of proteoglycans in the group of autograft implants [8].

Apoptosis contributes to meniscal injury [3]. Our observations regarding excessive apoptosis and the changes of the biochemical and mechanical properties of implants may explain the increased susceptibility of the implants to injury when compared to natural menisci $[9,10]$.

We do not link excessive apoptosis in transplanted menisci with revascularization. Firstly revascularization of the meniscal graft starts in the first weeks after transplantation [7, 21]. Our studies were done 6 months after transplantation when the menisci had healed completely and the graft was in a state of advanced rebuilding. Secondly the revascularization takes place in the peripheral part of the graft only. We assessed the apoptosis in the posterior part of grafted menisci both in the peripheral and central part of the transplant (with no blood vessels). Hashimoto et al. showed a direct relationship between increased NO production and excessive apoptosis in degenerated menisci [2].

Our immunohistochemical analyses demonstrated a low level of collagenase-1 and stromelysin-1 expression in the control group and in the group of autografts, which suggests that both metalloproteinases take part in extracellular matrix changes in the natural and implanted meniscus. Increased expression of MMP-1 and MMP-3 in allografts points to active remodelling of the extracellular matrix and to irreversible alterations in its compo-

Table II. Water, total collagen and GAGs content in control and study groups

\begin{tabular}{|lccc|}
\hline Group & Water [\%] & Total collagen $[\mu \mathrm{g} / \mathrm{mg}$ dry mass] & GAGs $[\mu \mathrm{g} / \mathrm{mg}$ dry mass] \\
\hline Control & $73.48 \pm 0.98$ & $818.77 \pm 11.73$ & $94.74 \pm 2.59$ \\
\hline Autografts & $77.06 \pm 0.80^{\infty}$ & $815.18 \pm 10.99$ & $80.58 \pm 3.19^{\infty}$ \\
\hline Allografts & $84.07 \pm 1.03^{*} \&$ & $704.80 \pm 18.75^{*} \&$ & $56.92 \pm 1.62^{*} \&$ \\
\hline
\end{tabular}

Mean value \pm standard deviation (SD). ${ }^{\infty}$ Statistically significant between control and autografts $(p<0.05)$. *Statistically significant between control and allografts $(p<0.05)$. \&Statistically significant between auto- and allografts $(p<0.05)$ 
sition, which is confirmed by the statistically significant decrease of total collagen content in our studies on allografts [18]. An immunohistochemical analysis demonstrated slight TIMP-2 expression in the menisci of the control group and in autografts (no statistical differences), indicating its participation in the regulation of extracellular matrix changes in natural menisci and fresh grafts by similar regulation of metalloproteinase activity. The increased TIMP-2 and MMP-3 expression in allografts confirms the complex nature of homeostasis disorders in allograft implants. Stromelysin-1 not only takes part in the degradation of proteoglycans, which enables collagen lysis by collagenase, but can also participate in the alternative path of pro-MMP-9 activation [18, 30].

The limitations of our study are caused by the fact that the obtained results are based on a rabbit experimental model. The semi-quantitative methodology of immunohistochemical analyses and lack of TIMP-1 determination are also limitations. The results of the immunohistochemical, mechanical and biochemical tests are limited to the posterior third of the meniscus. The small size of the menisci forced a simplification of the mechanical tests.

In conclusion, the presence of excessive apoptosis in both kinds of meniscal grafts (auto- and allografts) suggests that apoptosis can be a primary phenomenon independent of the kind of graft, having unbeneficial prognostic importance. Further studies should elucidate the role of factors inducing apoptosis and assess the possibilities of its inhibition.

Our results suggest that apoptosis and increased MMP-1 and MMP-3 expression have an adverse effect on the biological properties of implants.

The clinical relevance of the study is to show excessive apoptosis in both meniscal grafts - autoand allografts. It was demonstrated that excessive apoptosis was accompanied by detrimental changes in the mechanical and biochemical properties crucial for the fate of the grafts $[7,29]$. The future strategy of using allografts in humans is designing a method of apoptosis inhibition in implanted menisci to improve long-term outcomes.

\section{Acknowledgments}

This study was supported by a research project of the Medical University of Lodz 502-17-263.

\section{References}

1. Cole BJ, Carter TR, Rodeo SA. Allograft meniscal transplantation. Background, techniques and results. J Bone Joint Surg Am 2002; 84: 1236-50.

2. Hashimoto S, Takahashi K, Ochs RL, Coutts RD, Amiel D, Lotz M. Nitric oxide production and apoptosis in cells of the meniscus during experimental osteoarthritis. Arthritis Rheum 1999; 42: 2123-31.

3. Le Graverand HMP, Vegnon E, Otterness IG, Hart DA. Early changes in lapine menisci during osteoarthritis development. Part I: Cellular and matrix alteration. Osteoarthritis Cart 2001; 9: 56-64.

4. Le Graverand HMP, Vegnon E, Otterness IG, Hart DA. Early changes in lapine menisci during osteoarthritis development. Part II: Mollecular alteration. Osteoarthritis Cart 2001; 9: 65-72.

5. Le Graverand HMP, Sciore P, Eggerer J, et al. Formation and phenotype of cell clusters in osteoarthitic meniscus. Arthtitis Rheum 2001; 44: 1808-18.

6. Robertson CM, Pennock AT, Harwood FL, Pomerleau BA, Allen RT, Amiel D. Characterization of pro-apoptotic and matrix-degenerative gene expression following induction of osteoarthritis in mature and aged rabbits. Osteoarthritis Cart 2006; 14: 471-6.

7. Arkel ERA, Boer HH. Human Maniscal transplantation. Preliminary results at 2 to 5-years follow-up. J Bone Jt Surg Br 1995; 77: 589-95.

8. Jackson DW, Mc Devitt CA, Simon TM, Arnoczky ST, Atwell EA, Silvino NJ. Meniscal transplantation using fresh and cryopreserved allografts. Am J Sports Med 1992; 20: 644-56.

9. Rath E, Richmond JC, Yassir W, Albright JD, Gundogan F. Meniscal allograft transplantation. Two to eight-year results. Am J Sports Med 2001; 29: 410-4.

10. Rodeo SA, Seneviratne A, Suzuki K, Felker K, Wickiewicz TL, Warren RF. Histological analysis of human meniscal allografts. A preliminary report. J Bone Jt Surg Am 2000; 82: 1071-82.

11. Stone KR, Rodkey WG, Kinney LA, Steadman JR. Autogenous replacement of the meniscus cartilage: analysis of results and mechanism of failure. Arthroscopy 1995; 11: 395-400.

12. Szomor ZL, Martin TE, Bonar F, Murrel GAC. The protecive effects of meniscal transplantation an cartilage. An experimental study in sheep. J. Bone Jt Surg Am 2000; 8: 80-8.

13. Majno G, Joris I. Apoptosis, oncosis and necrosis. An overview of cell death. Am J Pathol 1995; 146: 3-15.

14. Wyllie AH, Kerr JF, Currie AR. Cell death: the significance of apoptosis. Int Rev Cytol 1980; 68: 251-306.

15. Fu J, Wang P, Zhang X, et al. Myeloma cells inhibit osteogenic differentiation of mesenchymal cells and kill osteoblasts via TRAIL-induced apoptosis. Arch Med Sci 2010; 4: 496-504.

16. Visse R, Nagese H. Matrix metalloproteinases and tissue inhibitors of metalloproteinases. Structure, function and biochemistry. Circ Res 2003; 92: 827-39.

17. Woessner J Jr. Matrix metalloproteinases and inhibitors in connective tissue remodeling. FASEB J 1991; 5: 2145-54.

18. Dreier R, Grässel S, Fuchs S, Schaumburger J, Bruckner P. Pro-MMP-9 is a specific macrophage product and is activated by osteoarthritic chondrocytes via MMP-3 or MT1MMP/MMP-13 cascade. Exp Cell Res 2004; 297: 303-12.

19. Baker AH, Edwards DR, Murphy G. Metalloproteinase inhibitors: biologic actions and therapeutic opportunities. J Cell Sci 2002; 115: 3719-27.

20. Verdonk R. Alternative treatments for meniscal injuries. J Bone Joint Surg Br 1997; 79: 866-73.

21. Shibuya S. Meniscus transplantation using a cryopreserved allograft. Histological and ultrastructural study of the transplantated meniscus. J Orthop Sci 1999; 4: 135-141.

22. Sommerlath K, Gillquist J. The effect of an artificial meniscus substitute in a knee joint with a resected 
anterior cruciate ligament. An experimental study in rabbits. Clin Orthop 1993; 289: 276-84.

23. Durand E, Scoazec A, Lafont A, et al. In vivo induction of endothelial apoptosis leads to vessel thrombosis and endothelial denudation. A clue to the understanding of the mechanisms of thromotic plaque erosion. Circulation 2004; 109: 2503-6.

24. Choi HR, Kondo S, Hirose K, Ishiguro N, Hasegawa $Y$, Iwata $\mathrm{H}$. Expression and enzymatic activity of MMP-2 during healing process of the acute supraspinatus tendon tear in rabbits. J Orthop Res 2002; 20: 927-33.

25. DeHaven KE. The role of the meniscus. In: Articular cartilage and knee joint function: basic science and arthroscopy. Ewing JW (ed). Raven, New York 1990; 103-15.

26. Woessner JF Jr. The determination of hydroxyproline in tissue and protein samples containing small portions of this amino acid. Arch Biochem Biophys 1961; 93: 440-7.

27. Ingman AM, Ghosh P, Taylor TKF. Variation of collagenuos and non-collagenous proteins of human menisci with age and degeneration. Gerontologia 1974; 20: 212-23.

28. Fernale RW, Buttle DJ, Barrett AJ. Improved quantitation and discrimination of sulfated glycosaminoglycans by use dimethylmethylene blue. Biochem Biophys Acta 1986; 883: 173-7.

29. de Boer HH, Koudstaal J. Failed meniscus transplantation. A report of three cases. Clin Orthop 1994; 306: 155-62.

30. Tsuzaki M, Guyton G, Garret W, et al. IL-1beta induced COX2, MMP-1,3 and 13, ADAMTS-4, IL-1 beta and II- 6 in human tendon cells. J Orthop Res 2003; 21: 256-64. 